Asia Pac. J. Math. 2021 8:18

\title{
ENTROPY SOLUTIONS FOR SOME NONLINEAR ELLIPTIC PROBLEM VIA MINTY'S LEMMA IN MUSIELAK-ORLICZ-SOBOLEV SPACES
}

\author{
BADR EL HAJI ${ }^{1, *}$, MOHAMED MABDAOUI ${ }^{2}$
}

${ }^{1}$ Laboratory LaR2A, Mathematics Department, Faculty of Sciences of Tetouan, Abdelmalek Essaadi University, BP 2121, Tetouan, Morocco

${ }^{2}$ Sidi bennour Polydisciplinary Faculty , University Chouaib Doukkali, P.O. Box 20, 24000 El Jadida, Morocco

*Corresponding author: badr.elhaji@gmail.com

Received Apr. 15, 2021

AвstRACt. We prove an existence result of entropy solution for a class of nonlinear elliptic problems of Leray-Lions type with large monotonicity condition in the framework of MusielakOrlicz-Sobolev spaces and with right hand side $f \in L^{1}(\Omega)$ and $F \in\left(C^{0}(\mathbb{R})\right)^{N}$.

2010 Mathematics Subject Classification. 46E35, 35K60, 35J20, 35J25, 35J60.

Key words and phrases. elliptic problem; entropy solutions; Musielak-Orlicz-Sobolev spaces; compact imbedding; $\Delta_{2}$-condition.

\section{InTRODUCTION}

This paper deals with existence of solutions to the following nonlinear Dirichlet problem

$$
\begin{cases}A(u)+g(x, u)=f(x)-\operatorname{div} F(u) & \text { in } \quad \Omega, \\ u=0 & \text { on } \partial \Omega,\end{cases}
$$

where $A(u)=-\operatorname{div}(a(x, u, \nabla u)), \Omega$ is a bounded domain of $\mathbb{R}^{N}, N \geq 2$. $a(x, u, \nabla u)=$ $\left(a_{i}(x, u, \nabla u)\right)_{1 \leq i \leq N}, a_{i}: \Omega \times \mathbb{R} \times \mathbb{R}^{N} \rightarrow \mathbb{R}$ is a Carathéodory functions (that is measurable with respect to $x$ in $\Omega$ for every $(s, \xi) \in \mathbb{R} \times \mathbb{R}^{N}$, and continuous with respect to $(s, \xi) \in \mathbb{R} \times \mathbb{R}^{N}$ for almost every $x \in \Omega$ ) such that for all $\xi, \xi^{\prime}$ in $\mathbb{R}^{N}$,

$$
\left|a_{i}(x, s, \xi)\right| \leq\left|\phi_{i}(x)\right|+K_{i} \bar{P}^{-1}\left(\varphi\left(x, c_{2}|s|\right)\right)+K_{i}\left(\bar{\varphi}^{-1} \varphi\left(x, c_{1}|\xi|\right)\right),
$$

DOI: $10.28924 / \mathrm{APJM} / 8-18$ 


$$
\left(a(x, s, \xi)-a\left(x, s, \xi^{\prime}\right)\right)\left(\xi-\xi^{\prime}\right) \geq 0
$$

$$
a(x, s, \xi) \xi \geq \alpha \varphi\left(x, \lambda_{1}|\xi|\right)
$$

where $c_{1}, c_{2}, \lambda_{1}$ and $K_{i}$ belongs to $\mathbb{R}_{+} \cdot \varphi, P$ be two Musielak functions such that $P \prec \prec \varphi$. Moreover $\bar{\varphi}, \bar{P}$ be the complementary functions of $\varphi$ and $P$ respectively, and $\phi_{i} \in E_{\bar{\varphi}}(\Omega)$.

Moreover, the function $g(x, s)$ is a Caratheodory function satisfying

$$
\begin{gathered}
g(x, s) s \geq 0, \\
\sup _{|s| \leq n}|g(x, s)|=g_{n}(x) \in L^{1}(\Omega),
\end{gathered}
$$

In [14], Boccardo has proved the existence of entropy solutions and some regularity results for degenerate elliptic problem whose model is

$$
\begin{cases}-\operatorname{div} a(x, u, \nabla u)=f-\operatorname{div} \phi(u) & \text { in } \Omega \\ u=0 & \text { on } \partial \Omega\end{cases}
$$

with $f \in L^{1}(\Omega)$ and $\phi(\cdot) \in C^{0}\left(R, R^{N}\right)$.

In [13], the following quasilinear elliptic problem

$$
\left\{\begin{array}{cl}
-\operatorname{div} a(x, u, \nabla u)=f-\operatorname{div} F & \text { in } \Omega \\
u=0 & \text { on } \partial \Omega
\end{array}\right.
$$

where $a(x, s, \xi)$ is a Caratheodory function, $f \in L^{1}(\Omega)$ and $F \in\left(L^{p^{\prime}}(\Omega)\right)^{N}$, have been studied by Boccardo and Orsina where they have proved the existence result, by introducing an $L^{1}-$ version of Minty's Lemma.

In the general framework of weighted Orlicz-Sobolev spaces, El haji, El Moumni and Kouhaila ( [22]), have proved an existence solutions to the following nonlinear Dirichlet problem

$$
\begin{cases}-\operatorname{div} a(x, u, \nabla u)=f(x) & \text { in } \quad \Omega, \\ u=0 & \text { on } \partial \Omega\end{cases}
$$


with $f \in L^{1}$.

In the framework of weighted Sobolev spaces, Akdim, Azroul and Rhoudaf have proved in [2] the existence of $T$ - solutions for the elliptic problem

$$
\left\{\begin{array}{cc}
-\operatorname{div} a(x, u, \nabla u)=F & \text { in } \Omega \\
u=0 & \text { on } \partial \Omega .
\end{array}\right.
$$

where $F \in W^{-1, p^{\prime}}\left(\Omega, \omega^{*}\right)$, and only large monotonicity is assumed on the Caratheodory function $a(x, u, \nabla u)$.

For the case of Orlicz spaces, Gossez and Mustonen [26] have studied the unilateral elliptic problem associated to the following equation

$$
A(u)+g(x, u)=f \quad \text { in } \quad \Omega,
$$

See also [4] for the anisotropic case, and [3] for the case of variable exponent.

Recently, much attention has been paid to the existence of solutions of elliptic and parabolic problems under various assumptions; see, e.g., $[11,12,17,18,20,21,23,33-36,38,39]$ and the references therein.

The feature of this paper, is to treat a class of problems for which the classical monotone operator methods (developed by Minty [31], Browder [16], Brézis [15] and Lions [30] in $W_{0}^{1, p}(\Omega)$ case $)$ do not apply. The reason for this, is that $a(x, u, \nabla u)$ does not need to satisfy the strict monotonicity condition that is,

$$
\left(a(x, s, \xi)-a\left(x, s, \xi^{\prime}\right)\right)\left(\xi-\xi^{\prime}\right)>0, \text { for all } \xi, \xi^{\prime} \in \mathbb{R}^{N},\left(\xi \neq \xi^{\prime}\right),
$$

of a typical Leray-Lions operator but only a large monotonicity that is

$$
\left(a(x, s, \xi)-a\left(x, s, \xi^{\prime}\right)\right)\left(\xi-\xi^{\prime}\right) \geq 0, \text { for all } \xi, \xi^{\prime} \in \mathbb{R}^{N},
$$

The aim of this paper is to demonstrate the existence of solutions for (1.1) under the weaker assumption large monotonicity condition, without using the almost everywhere convergence of the gradients of the approximate equations, since this is impossible to prove in our setting. Our proof is based on a version of Minty's Lemma .

The paper is organized as follows: In section 2, we introduce some basic definitions and properties in the setting of Musielak-Orlicz-Sobolev spaces and we prepare some auxiliary results to prove our theorem. In the final section 3, we state the main result and proofs. 


\section{Preliminary}

Here we give some definitions and properties that concern Musielak-Orlicz spaces (see [32]). Let $\Omega$ be an open subset of $\mathbb{R}^{n}$, a Musielak-Orlicz function $\varphi$ is a real-valued function defined in $\Omega \times \mathbb{R}^{+}$such that

a) $\varphi(x,$.$) is an N$-function for all $x \in \Omega$ (i.e. convex, nondecreasing, continuous, $\varphi(x, 0)=$ $0, \varphi(x, t)>0$ for all $t>0$ and $\lim _{t \rightarrow 0} \sup _{x \in \Omega} \frac{\varphi(x, t)}{t}=0$ and $\left.\lim _{t \rightarrow \infty} \inf _{x \in \Omega} \frac{\varphi(x, t)}{t}=\infty\right)$.

b) $\varphi(., t)$ is a measurable function for all $t \geq 0$.

For a Musielak-Orlicz function $\varphi$, let $\varphi_{x}(t)=\varphi(x, t)$ and let $\varphi_{x}^{-1}$ be the nonnegative reciprocal function with respect to $t$, i.e. the function that satisfies

$$
\varphi_{x}^{-1}(\varphi(x, t))=\varphi\left(x, \varphi_{x}^{-1}(t)\right)=t
$$

The Musielak-Orlicz function $\varphi$ is said to satisfy the $\Delta_{2}$-condition if for some $k>0$, and a nonnegative function $h$, integrable in $\Omega$, we have

$$
\varphi(x, 2 t) \leq k \varphi(x, t)+h(x) \text { for all } x \in \Omega \text { and } t \geq 0 .
$$

When (2.1) holds only for $t \geq t_{0}>0$, then $\varphi$ is said to satisfy the $\Delta_{2}$-condition near infinity. Let $\varphi$ and $\gamma$ be two Musielak-Orlicz functions, we say that $\varphi$ dominate $\gamma$ and we write $\gamma \prec \varphi$, near infinity (resp. globally) if there exist two positive constants $c$ and $t_{0}$ such that for a.e. $x \in \Omega$ :

$$
\left.\gamma(x, t) \leq \varphi(x, c t) \text { for all } t \geq t_{0}, \text { (resp. for all } t \geq 0 \text { i.e. } t_{0}=0\right) .
$$

We say that $\gamma$ grows essentially less rapidly than $\varphi$ at 0 (resp. near infinity) and we write $\gamma \prec \prec \varphi$ if for every positive constant $c$ we have

$$
\lim _{t \rightarrow 0}\left(\sup _{x \in \Omega} \frac{\gamma(x, c t)}{\varphi(x, t)}\right)=0, \quad\left(\text { resp. } \lim _{t \rightarrow \infty}\left(\sup _{x \in \Omega} \frac{\gamma(x, c t)}{\varphi(x, t)}\right)=0\right) .
$$

For a Musielak-Orlicz function $\varphi$ and a measurable function $u: \Omega \longrightarrow \mathbb{R}$, we define the functional

$$
\rho_{\varphi, \Omega}(u)=\int_{\Omega} \varphi(x,|u(x)|) d x .
$$

The set $K_{\varphi}(\Omega)=\left\{u: \Omega \longrightarrow \mathbb{R}\right.$ measurable $\left./ \rho_{\varphi, \Omega}(u)<\infty\right\}$ is called as the Musielak-Orlicz class (or generalized Orlicz class). The Musielak-Orlicz space (the generalized Orlicz spaces) $L_{\varphi}(\Omega)$ is the vector space generated by $K_{\varphi}(\Omega)$, that is, $L_{\varphi}(\Omega)$ is the smallest linear space containing the set $K_{\varphi}(\Omega)$. Equivalently

$$
L_{\varphi}(\Omega)=\left\{u: \Omega \longrightarrow \mathbb{R} \text { measurable } / \rho_{\varphi, \Omega}\left(\frac{u}{\lambda}\right)<\infty, \text { for some } \lambda>0\right\} .
$$


For a Musielak-Orlicz function $\varphi$ we put:

$$
\psi(x, s)=\sup _{t>0}\{s t-\varphi(x, t)\}
$$

Note that $\psi$ is the Musielak-Orlicz function complementary to $\varphi$ (or conjugate of $\varphi$ ) in the sense of Young with respect to the variable $s$. In the space $L_{\varphi}(\Omega)$ we define the following two norms:

$$
\|u\|_{\varphi, \Omega}=\inf \left\{\lambda>0 / \int_{\Omega} \varphi\left(x, \frac{|u(x)|}{\lambda}\right) d x \leq 1\right\},
$$

which is called the Luxemburg norm and the so-called Orlicz norm by:

$$
\|u\|_{\varphi, \Omega}=\sup _{\|v\|_{\psi} \leq 1} \int_{\Omega}|u(x) v(x)| d x
$$

where $\psi$ is the Musielak-Orlicz function complementary to $\varphi$. These two norms are equivalent (see [32]). The closure in $L_{\varphi}(\Omega)$ of the bounded measurable functions with compact support in $\bar{\Omega}$ is denoted by $E_{\varphi}(\Omega)$, it is a separable space (see [32], Theorem 7.10).

We say that sequence of functions $u_{n} \in L_{\varphi}(\Omega)$ is modular convergent to $u \in L_{\varphi}(\Omega)$ if there exists a constant $\lambda>0$ such that

$$
\lim _{n \rightarrow \infty} \rho_{\varphi, \Omega}\left(\frac{u_{n}-u}{\lambda}\right)=0 .
$$

For any fixed nonnegative integer $m$ we define

$$
W^{m} L_{\varphi}(\Omega)=\left\{u \in L_{\varphi}(\Omega) / \forall|\alpha| \leq m, D^{\alpha} u \in L_{\varphi}(\Omega)\right\}
$$

and

$$
W^{m} E_{\varphi}(\Omega)=\left\{u \in E_{\varphi}(\Omega) / \forall|\alpha| \leq m, D^{\alpha} u \in E_{\varphi}(\Omega)\right\}
$$

where $\alpha=\left(\alpha_{1}, \ldots, \alpha_{n}\right)$ with nonnegative integers $\alpha_{i},|\alpha|=\left|\alpha_{1}\right|+\ldots+\left|\alpha_{n}\right|$ and $D^{\alpha} u$ denote the distributional derivatives. The space $W^{m} L_{\varphi}(\Omega)$ is called the Musielak-Orlicz Sobolev space. Let for $u \in W^{m} L_{\varphi}(\Omega)$ :

$$
\bar{\rho}_{\varphi, \Omega}(u)=\sum_{|\alpha| \leq m} \rho_{\varphi, \Omega}\left(D^{\alpha} u\right) \text { and }\|u\|_{\varphi, \Omega}^{m}=\inf \left\{\lambda>0 / \bar{\rho}_{\varphi, \Omega}\left(\frac{u}{\lambda}\right) \leq 1\right\},
$$

these functionals are a convex modular and a norm on $W^{m} L_{M}(\Omega)$, respectively, and the pair $\left(W^{m} L_{\varphi}(\Omega),\|\cdot\|_{\varphi, \Omega}^{m}\right)$ is a Banach space if $\varphi$ satisfies the following condition (see [32]):

$$
\text { There exist a constant } c_{0}>0 \text { such that } \inf _{x \in \Omega} \varphi(x, 1) \geq c_{0} .
$$

The space $W^{m} L_{\varphi}(\Omega)$ will always be identified to a subspace of the product $\prod_{|\alpha| \leq m} L_{\varphi}(\Omega)=$ $\Pi L_{\varphi}$, this subspace is $\sigma\left(\Pi L_{\varphi}, \Pi E_{\psi}\right)$ closed. 
The space $W_{0}^{m} L_{\varphi}(\Omega)$ is defined as the $\sigma\left(\Pi L_{\varphi}, \Pi E_{\psi}\right)$ closure of $\mathcal{D}(\Omega)$ in $W^{m} L_{\varphi}(\Omega)$, and the space $W_{0}^{m} E_{\varphi}(\Omega)$ as the (norm) closure of the Schwartz space $\mathcal{D}(\Omega)$ in $W^{m} L_{\varphi}(\Omega)$.

Let $W_{0}^{m} L_{\varphi}(\Omega)$ be the $\sigma\left(\Pi L_{\varphi}, \Pi E_{\psi}\right)$ closure of $\mathcal{D}(\Omega)$ in $W^{m} L_{\varphi}(\Omega)$. The following spaces of distributions will also be used:

$$
W^{-m} L_{\psi}(\Omega)=\left\{f \in \mathcal{D}^{\prime}(\Omega) / f=\sum_{|\alpha| \leq m}(-1)^{|\alpha|} D^{\alpha} f_{\alpha} \text { with } f_{\alpha} \in L_{\psi}(\Omega)\right\}
$$

and

$$
W^{-m} E_{\psi}(\Omega)=\left\{f \in \mathcal{D}^{\prime}(\Omega) / f=\sum_{|\alpha| \leq m}(-1)^{|\alpha|} D^{\alpha} f_{\alpha} \text { with } f_{\alpha} \in E_{\psi}(\Omega)\right\} .
$$

We say that a sequence of functions $u_{n} \in W^{m} L_{\varphi}(\Omega)$ is modular convergent to $u \in W^{m} L_{\varphi}(\Omega)$ if there exists a constant $k>0$ such that

$$
\lim _{n \rightarrow \infty} \bar{\rho}_{\varphi, \Omega}\left(\frac{u_{n}-u}{k}\right)=0 .
$$

We recall that

$$
\varphi(x, t) \leq t \psi^{-1}(\varphi(x, t)) \leq 2 \varphi(x, t) \quad \text { for all } t \geq 0
$$

For $\varphi$ and her complementary function $\psi$, the following inequality is called the Young inequality (see [32]):

$$
t s \leq \varphi(x, t)+\psi(x, s), \quad \forall t, s \geq 0, \text { a.e. } x \in \Omega
$$

This inequality implies that

$$
\|u\|_{\varphi, \Omega} \leq \rho_{\varphi, \Omega}(u)+1
$$

In $L_{\varphi}(\Omega)$ we have the relation between the norm and the modular

$$
\|u\|_{\varphi, \Omega} \leq \rho_{\varphi, \Omega}(u) \quad \text { if }\|u\|_{\varphi, \Omega}>1
$$

and

$$
\|u\|_{\varphi, \Omega} \geq \rho_{\varphi, \Omega}(u) \quad \text { if }\|u\|_{\varphi, \Omega} \leq 1
$$

For two complementary Musielak-Orlicz functions $\varphi$ and $\psi$, let $u \in L_{\varphi}(\Omega)$ and $v \in L_{\psi}(\Omega)$, then we have the Hölder inequality (see [32]):

$$
\left|\int_{\Omega} u(x) v(x) d x\right| \leq\|u\|_{\varphi, \Omega}\|v\|_{\psi, \Omega} .
$$


Def 2.1. A Musielak function $\varphi$ is called locally integrable on $\Omega$ if

$$
\int_{E} \varphi(x, t) d x=\int_{\Omega} \varphi\left(x, t \chi_{E}(x)\right) d x<+\infty
$$

for all $t \geq 0$ and all measurable set $E \subset \Omega$ with $\operatorname{mes}(E)<+\infty$.

Remark 1. If $P \prec \prec \varphi$ near infinity such that $P$ is locally integrable on $\Omega$, then $\forall c>0$ there exists a nonnegative integrable function $h$ such that

$$
P(x, t) \leq \varphi(x, c t)+h(x), \text { for all } t \geq 0 \text { and for a.e. } x \in \Omega \text {. }
$$

Def 2.2. A Musielak function $\varphi$ satisfies the log-Hölder continuity condition on $\Omega$ if there exists a constant $A>0$ such that

$$
\frac{\varphi(x, t)}{\varphi(y, t)} \leq t^{\left(\frac{A}{\log \left(\frac{1}{|x-y|}\right)}\right)}
$$

for all $t \geq 1$ and for all $x, y \in \Omega$ with $|x-y| \leq \frac{1}{2}$.

We will also use the following technical lemmas.

2.1. Some technical lemmas. We will use the following technical lemmas.

Lemma 2.1. [5] Let $\Omega$ be a bounded Lipschitz domain in $\mathbb{R}^{N}(N \geq 2)$ and let $\varphi$ be a Musielak function satisfying the log-Hölder continuity such that

$$
\bar{\varphi}(x, 1) \leq c_{1} \quad \text { a.e in } \Omega \text { for some } c_{1}>0 \text {. }
$$

Then $D(\Omega)$ is dense in $L_{\varphi}(\Omega)$ and in $W_{0}^{1} L_{\varphi}(\Omega)$ for the modular convergence.

Remark 2. Note that if $\lim _{t \rightarrow \infty} \inf _{x \in \Omega} \frac{\varphi(x, t)}{t}=\infty$, then (2.9) holds:

Example 2.1. Let $p \in P(\Omega)$ a bounded variable exponent on $\Omega$, such that there exists a constant $A>0$ such that for all points $x, y \in \Omega$ with $|x-y|<\frac{1}{2}$, we have the inequality

$$
|p(x)-p(y)| \leq \frac{A}{\log \left(\frac{1}{|x-y|}\right)}
$$

We can verify that the Musielak function defined by $\varphi(x, t)=t^{p(x)} \log (1+t)$ satisfies the conditions of Lemma 2.1. 
Lemma 2.2. [5] (Poincare's inequality: Integral form) Let $\Omega$ be a bounded Lipschitz domain of $\mathbb{R}^{N}(N \geq 2)$ and let $\varphi$ be a Musielak function satisfying the conditions of Lemma 2.1. Then there exists positive constants $\beta, \eta$ and $\lambda$ depending only on $\Omega$ and $\varphi$ such that

$$
\int_{\Omega} \varphi(x,|v|) d x \leq \beta+\eta \int_{\Omega} \varphi(x, \lambda|\nabla v|) d x \text { for all } v \in W_{0}^{1} L_{\varphi}(\Omega) .
$$

Corollary 2.3. [5] (Poincare's inequality) Let $\Omega$ be a bounded Lipschitz domain of $\mathbb{R}^{N}(N \geq 2$ ) and let $\varphi$ be a Musielak function satisfying the same conditions of Lemma2.2. Then there exists a constant $C>0$ such that

$$
\|v\|_{\varphi} \leq C\|\nabla v\|_{\varphi} \quad \forall v \in W_{0}^{1} L_{\varphi}(\Omega) .
$$

Lemma 2.4. [37] Let $F: \mathbb{R} \longrightarrow \mathbb{R}$ be uniformly Lipschitzian, with $F(0)=0$. Let $\varphi$ be a MusielakOrlicz function and let $u \in W_{0}^{1} L_{\varphi}(\Omega)$. Then $F(u) \in W_{0}^{1} L_{\varphi}(\Omega)$.

Moreover, if the set $D$ of discontinuity points of $F^{\prime}$ is finite, we have

$$
\frac{\partial}{\partial x_{i}} F(u)= \begin{cases}F^{\prime}(u) \frac{\partial u}{\partial x_{i}} & \text { a.e in }\{x \in \Omega: u(x) \in D\} \\ 0 & \text { a.e in }\{x \in \Omega: u(x) \notin D\} .\end{cases}
$$

Lemma 2.5. [9] Suppose that $\Omega$ satisfies the segment property and let $u \in W_{0}^{1} L_{\varphi}(\Omega)$. Then, there exists a sequence $\left(u_{n}\right) \subset \mathcal{D}(\Omega)$ such that

$$
u_{n} \rightarrow u \text { for modular convergence in } W_{0}^{1} L_{\varphi}(\Omega) .
$$

Furthermore, if $u \in W_{0}^{1} L_{\varphi}(\Omega) \cap L^{\infty}(\Omega)$ then $\left\|u_{n}\right\|_{\infty} \leq(N+1)\|u\|_{\infty}$.

Lemma 2.6. [27] Let $\left(f_{n}\right), f \in L^{1}(\Omega)$ such that

i) $f_{n} \geq 0$ a.e in $\Omega$,

ii) $f_{n} \longrightarrow f$ a.e in $\Omega$,

iii) $\int_{\Omega} f_{n}(x) d x \longrightarrow \int_{\Omega} f(x) d x$.

Then $f_{n} \longrightarrow f$ strongly in $L^{1}(\Omega)$.

Lemma 2.7. [10] If a sequence $g_{n} \in L_{\varphi}(\Omega)$ converges in measure to a measurable function $g$ and if $g_{n}$ remains bounded in $L_{\varphi}(\Omega)$, then $g \in L_{\varphi}(\Omega)$ and $g_{n} \rightarrow g$ for $\sigma\left(\Pi L_{\varphi}, \Pi E_{\psi}\right)$.

Lemma 2.8. [10] Let $u_{n}, u \in L_{\varphi}(\Omega)$. If $u_{n} \rightarrow u$ with respect to the modular convergence, then $u_{n} \rightarrow u$ for $\sigma\left(L_{\varphi}(\Omega), L_{\psi}(\Omega)\right)$.

Lemma 2.9. [24] If $P \prec \varphi$ and $u_{n} \rightarrow u$ for the modular convergence in $L_{\varphi}(\Omega)$, then $u_{n} \rightarrow u$ strongly in $E_{P}(\Omega)$. 
Lemma 2.10. [40] (Jensen inequality). Let $\varphi: \mathbb{R} \longrightarrow \mathbb{R}$ a convex function and $g: \Omega \longrightarrow \mathbb{R}$ is function measurable, then

$$
\varphi\left(\int_{\Omega} g d \mu\right) \leq \int_{\Omega} \varphi \circ g d \mu .
$$

Lemma 2.11. (The Nemytskii Operator). Let $\Omega$ be an open subset of $\mathbb{R}^{N}$ with finite measure and let $\varphi$ and $\psi$ be two Musielak Orlicz functions. Let $f: \Omega \times \mathbb{R}^{p} \longrightarrow \mathbb{R}^{q}$ be a Carathéodory function such that for a.e. $x \in \Omega$ and all $s \in \mathbb{R}^{p}$ :

$$
|f(x, s)| \leq c(x)+k_{1} \psi_{x}^{-1} \varphi\left(x, k_{2}|s|\right)
$$

where $k_{1}$ and $k_{2}$ are real positive constants and $c(\cdot) \in E_{\psi}(\Omega)$. Then the Nemytski Operator $N_{f}$ defined by $N_{f}(u)(x)=f(x, u(x))$ is continuous from

$$
\mathcal{P}\left(E_{M}(\Omega), \frac{1}{k_{2}}\right)^{p}=\prod\left\{u \in L_{M}(\Omega): d\left(u, E_{M}(\Omega)\right)<\frac{1}{k_{2}}\right\}
$$

into $\left(L_{\psi}(\Omega)\right)^{q}$ for the modular convergence. Furthermore if $c(\cdot) \in E_{\gamma}(\Omega)$ and $\gamma \prec \prec \psi$ then $N_{f}$ is strongly continuous from $\mathcal{P}\left(E_{M}(\Omega), \frac{1}{k_{2}}\right)^{p}$ to $\left(E_{\gamma}(\Omega)\right)^{q}$.

Def 2.3. [1] ( Segment property ) : A domain $\Omega$ is said to satisfy the segment property, if there exist a finite open covering $\{\theta\}_{i=1}^{k}$ of $\bar{\Omega}$ and a corresponding nonzero vectors $z_{i} \in R^{N}$ such that $\left(\bar{\Omega} \cap \theta_{i}\right)+t z_{i} \subset \Omega$ for all $t \in(0,1)$ and $i=1, \ldots, k$.

Lemma 2.12. [25] Suppose that $\Omega$ satisfies the segment property and let $u \in W_{0}^{1} L_{\varphi}(\Omega)$. Then, there exists a sequence $u_{n} \in D(\Omega)$ such that

$$
u_{n} \rightarrow u \text { for modular convergence in } W_{0}^{1} L_{\varphi}(\Omega) \text {. }
$$

Furthermore, if $u \in W_{0}^{1} L_{\varphi}(\Omega) \cap L^{\infty}(\Omega)$ then $\left\|u_{n}\right\|_{\infty} \leq(N+1)\|u\|_{\infty}$.

Lemma 2.13. Let $\Omega$ be a bounded open subset of $\mathbb{R}^{N}$ with the segment property. If $u \in\left(W_{0}^{1} L_{\varphi}(\Omega)\right)^{N}$ then $\int_{\Omega} \operatorname{div}(u) d x=0$.

Proof. Fix a vector $u=\left(u^{1}, \ldots, u^{N}\right) \in\left(W_{0}^{1} L_{\varphi}(\Omega)\right)^{N}$. since $W_{0}^{1} L_{\varphi}(\Omega)$ is the closure of $C_{0}^{\infty}(\Omega)$ in $W^{1} L_{\varphi}(\Omega)$, then each term $u^{i}$ can be approximated by a suitable sequence $u_{k}^{i} \in D(\Omega)$ such that $u_{k}^{i}$ converges to $u^{i}$ in $W_{0}^{1} L_{\varphi}(\Omega)$. Moreover, due to the fact that $u_{k}^{i} \in C_{0}^{\infty}(\Omega)$, then the Green formula gives

$$
\int_{\Omega} \frac{\partial u_{k}^{i}}{\partial x_{i}}=\int_{\partial \Omega} u_{k}^{i} \vec{n} d s=0
$$


On the other hand, $\frac{\partial u_{k}^{i}}{\partial x_{i}} \rightarrow \frac{\partial u^{i}}{\partial x_{i}}$ in $L_{\varphi}(\Omega)$. Thus $\frac{\partial u_{k}^{i}}{\partial x_{i}} \rightarrow \frac{\partial u^{i}}{\partial x_{i}}$ in $L^{1}(\Omega)$, which gives in view of $(2.11)$ that

$$
\int_{\Omega} \operatorname{div}(u) d x=0
$$

Throughout the paper, $T_{k}$ denotes the truncation function at height $k \geq 0$ :

$$
T_{k}(s)=\max (-k, \min (k, s))
$$

\section{MAin Existence Theorem}

Let $Y$ be a closed subspace of $W^{1} L_{\varphi}(\Omega)$ for $\sigma\left(\prod L_{\varphi}, \prod E_{\bar{\varphi}}\right)$ and let

$$
Y_{0}=Y \cap W^{1} L_{\varphi}(\Omega),
$$

such that $Y$ is the closure of $Y_{0}$ for $\sigma\left(\prod L_{\varphi}, \prod E_{\bar{\varphi}}\right)$. In the next, we consider the complementary system $\left(Y, Y_{0}, Z, Z_{0}\right)$ generated by $Y$ i.e. $Y_{0}^{*}$ can be identified to $Z$ and $Z_{0}^{*}$ can be identified to $Y$ by the means $\langle.,$.$\rangle . Let the mapping T$ (associated to the operator $A$ ) defined from $D(T)=\left\{u \in Y, a_{0}(x, u, \nabla u) \in L_{\bar{\varphi}}(\Omega), a_{i}(x, u, \nabla u) \in L_{\bar{\varphi}}(\Omega)\right\}$ into $Z$ by the formula

$$
a(u, v)=\int_{\Omega} a_{0}(x, u, \nabla u) v(x) d x+\sum_{1 \leq i \leq N} \int_{\Omega} a_{i}(x, u, \nabla u) \frac{\partial v(x)}{\partial x_{i}} d x \quad \forall v \in Y_{0} .
$$

We consider the complementary system

$$
\left(Y, Y_{0}, Z, Z_{0}\right)=\left(W_{0}^{1} L_{\varphi}(\Omega), W_{0}^{1} E_{\varphi}(\Omega), W^{-1} E_{\bar{\varphi}}(\Omega), W^{-1} L_{\bar{\varphi}}(\Omega)\right)
$$

As in [14], we define the entropy solution of our problem.

Def 3.1. An entropy solution of the problem (1.1) is a measurable function $u$ such that $T_{k}(u) \in$ $W_{0}^{1} L_{\varphi}(\Omega)$ for every $k>0$ and such that

$$
\int_{\Omega} a(x, u, \nabla u) \nabla T_{k}(u-\phi) d x+\int_{\Omega} h(x, u) T_{k}(u-\phi) d x \leq \int_{\Omega} f T_{k}(u-\phi) d x+\int_{\Omega} F(u) \nabla T_{k}(u-\phi)
$$

for every $\phi \in W_{0}^{1} E_{\varphi}(\Omega) \cap L^{\infty}(\Omega)$.

Our main results are collected in the following theorem.

Theorem 3.1. Under the assumptions (1.2)-(1.7), there exist an entropy solution $u$ of the problem (1.1). 


\subsection{Main Lemma.}

Lemma 3.2. Let $u$ be a mesurable function such that $T_{k}(u)$ belongs to $W_{0}^{1} L_{\varphi}(\Omega)$ for every $k>0$. Then

$$
\int_{\Omega} a(x, u, \nabla \phi) \nabla T_{k}(u-\phi) d x \leq \int_{\Omega} f T_{k}(u-\phi) d x+\int_{\Omega} F(u) \nabla T_{k}(u-\phi)
$$

is equivalent to

$\int_{\Omega} a(x, u, \nabla u) \nabla T_{k}(u-\phi) d x+\int_{\Omega} g(x, u) T_{k}(u-\phi) d x=\int_{\Omega} f T_{k}(u-\phi) d x+\int_{\Omega} F(u) \nabla T_{k}(u-\phi)$ for every $\phi \in W_{0}^{1} L_{\varphi}(\Omega)$, and for every $k>0$.

3.2. Proof of lemma 3.2. In fact (3.2) implies (3.1) is easily proved adding and subtracting

$$
\int_{\Omega} a(x, u, \nabla \phi) \nabla T_{k}(u-\phi) d x,
$$

and then using assumption (1.3). Thus, it remains to prove that (3.1) implies (3.2). Let $h$ and $k$ be positive real numbers, let $\lambda \in]-1,1\left[\right.$ and $\Psi \in W_{0}^{1} L_{\varphi}(\Omega) \cap L^{\infty}(\Omega)$.

choose, $\phi=T_{h}\left(u-\lambda T_{k}(u-\Psi)\right) \in W_{0}^{1} L_{\varphi}(\Omega)$ as test function in (3.1), we have :

$$
I_{h k} \leq J_{h k}
$$

with

$$
\begin{aligned}
I_{h k}=\int_{\Omega} a\left(x, u, \nabla T_{h}\left(u-\lambda T_{k}(u-\Psi)\right)\right) & \nabla T_{k}\left(u-T_{h}\left(u-\lambda T_{k}(u-\Psi)\right)\right) d x \\
& +\int_{\Omega} g(x, u) T_{k}\left(u-T_{h}\left(u-\lambda T_{k}(u-\psi)\right)\right) d x=I_{h k}^{\prime}+I_{h k k}^{\prime \prime}
\end{aligned}
$$

and

$$
J_{h k}=\int_{\Omega} f T_{k}\left(u-T_{h}\left(u-\lambda T_{k}(u-\Psi)\right)\right) d x+\int_{\Omega} F(u) \nabla T_{k}\left(u-T_{h}\left(u-\lambda T_{k}(u-\Psi)\right)\right) .
$$

Put

$$
A_{h k}=\left\{x \in \Omega,\left|u-T_{h}\left(u-\lambda T_{k}(u-\Psi)\right)\right| \leq k\right\}
$$

and

$$
B_{h k}=\left\{x \in \Omega,\left|u-\lambda T_{k}(u-\Psi)\right| \leq h\right\}
$$


Then, we obtain

$$
\begin{aligned}
I_{h k}^{\prime}=\int_{A_{k h} \cap B_{h k}} a\left(x, u, \nabla T_{h}\left(u-\lambda T_{k}(u-\Psi)\right)\right) \nabla T_{k}\left(u-T_{h}\left(u-\lambda T_{k}(u-\Psi)\right)\right) d x \\
\quad+\int_{A_{k h} \cap B_{h k}^{C}} a\left(x, u, \nabla T_{h}\left(u-\lambda T_{k}(u-\Psi)\right)\right) \nabla T_{k}\left(u-T_{h}\left(u-\lambda T_{k}(u-\Psi)\right)\right) d x \\
\quad+\int_{A_{k h}^{C}} a\left(x, u, \nabla T_{h}\left(u-\lambda T_{k}(u-\Psi)\right)\right) \nabla T_{k}\left(u-T_{h}\left(u-\lambda T_{k}(u-\Psi)\right)\right) d x .
\end{aligned}
$$

Since $\nabla T_{k}\left(u-T_{h}\left(u-\lambda T_{k}(u-\Psi)\right)\right)$ is different to zero only on $A_{k h}$, we have

$$
\int_{A_{k h}^{C}} a\left(x, u, \nabla T_{h}\left(u-\lambda T_{k}(u-\Psi)\right)\right) \nabla T_{k}\left(u-T_{h}\left(u-\lambda T_{k}(u-\Psi)\right)\right) d x=0 .
$$

Moreover, if $x \in B_{h k}^{C}$, we have $\nabla T_{h}\left(u-\lambda T_{k}(u-\Psi)\right)=0$ and using (1.4), we deduce that,

$$
\begin{gathered}
\int_{A_{k h} \cap B_{h k}^{C}} a\left(x, u, \nabla T_{h}\left(u-\lambda T_{k}(u-\Psi)\right)\right) \nabla T_{k}\left(u-T_{h}\left(u-\lambda T_{k}(u-\Psi)\right)\right) d x \\
=\int_{A_{k h} \cap B_{h k}^{C}} a(x, u, 0) \nabla T_{k}\left(u-T_{h}\left(u-\lambda T_{k}(u-\Psi)\right)\right) d x=0 .
\end{gathered}
$$

From (3.4) and (3.5), we obtain

$$
I_{h k}=\int_{A_{k h} \cap B_{h k}} a\left(x, u, \nabla T_{h}\left(u-\lambda T_{k}(u-\Psi)\right)\right) \nabla T_{k}\left(u-T_{h}\left(u-\lambda T_{k}(u-\Psi)\right)\right) d x .
$$

Letting $h \rightarrow+\infty,|\lambda| \leq 1$, we have

$$
A_{k h} \rightarrow\left\{x,|\lambda|\left|T_{k}(u-\Psi)\right| \leq h\right\}=\Omega,
$$

$$
B_{h k} \rightarrow \Omega \quad \text { whichimplies } \quad A_{k h} \cap B_{h k} \rightarrow \Omega .
$$

Which and using Lebesgue theorem, we conclude that

thus implies that,

$$
\begin{aligned}
& \lim _{h \rightarrow+\infty} \int_{A_{k h} \cap B_{h k}} a\left(x, u, \nabla T_{h}\left(u-\lambda T_{k}(u-\Psi)\right)\right) \nabla T_{k}\left(u-T_{h}\left(u-\lambda T_{k}(u-\Psi)\right)\right) d x \\
& \quad=\lambda \int_{\Omega} a\left(x, u, \nabla\left(u-\lambda T_{k}(u-\Psi)\right)\right) \nabla T_{k}(u-\Psi) d x .
\end{aligned}
$$

$$
\lim _{h \rightarrow+\infty} I_{h k}^{\prime}=\lambda \int_{\Omega} a\left(x, u, \nabla\left(u-\lambda T_{k}(u-\Psi)\right)\right) \nabla T_{k}(u-\Psi) d x .
$$

moreover it is easy to see that

$$
\lim _{h \rightarrow+\infty} \int_{\Omega} g(x, u) T_{k}\left(u-T_{h}\left(u-\lambda T_{k}(u-\psi)\right)\right) d x=\lambda \int_{\Omega} g(x, u) T_{k}[u-\psi] d x
$$

thus implies that 
(3.9) $\lim _{h \rightarrow+\infty} I_{h k}=\lambda \int_{\Omega} a\left(x, u, \nabla\left(u-\lambda T_{k}(u-\Psi)\right)\right) \nabla T_{k}(u-\Psi) d x+\lambda \int_{\Omega} g(x, u) T_{k}[u-\psi] d x$.

On the other hand, we have,

$$
J_{h k}=\int_{\Omega} f T_{k}\left(u-T_{h}\left(u-\lambda T_{k}(u-\Psi)\right)\right) d x+\int_{\Omega} F(u) \nabla T_{k}\left(u-T_{h}\left(u-\lambda T_{k}(u-\Psi)\right)\right) d x .
$$

Then

$$
\begin{array}{r}
\lim _{h \rightarrow+\infty} \int_{\Omega} f T_{k}\left(u-T_{h}\left(u-\lambda T_{k}(u-\Psi)\right)\right) d x \\
+\int_{\Omega} F(u) \nabla T_{k}\left(u-T_{h}\left(u-\lambda T_{k}(u-\Psi)\right)\right) d x \\
=\lambda\left(\int_{\Omega} f T_{k}(u-\Psi) d x+\int_{\Omega} F(u) \nabla T_{k}(u-\Psi) d x\right),
\end{array}
$$

i.e.,

$$
\lim _{h \rightarrow+\infty} J_{h k}=\lambda\left(\int_{\Omega} f T_{k}(u-\Psi) d x+\int_{\Omega} F(u) \nabla T_{k}(u-\Psi) d x\right),
$$

Together (3.9), (3.13) and passing to the limit in (3.3), we obtain,

$$
\begin{array}{r}
\lambda\left(\int_{\Omega} a\left(x, u, \nabla\left(u-\lambda T_{k}(u-\Psi)\right)\right) \nabla T_{k}(u-\Psi) d x+\int_{\Omega} g(x, u) T_{k}[u-\psi] d x\right) \\
\leq \lambda\left(\int_{\Omega} f T_{k}(u-\Psi) d x+\int_{\Omega} F(u) \nabla T_{k}(u-\Psi) d x\right)
\end{array}
$$

for every $\Psi \in W_{0}^{1} L_{\varphi}(\Omega) \cap L^{\infty}(\Omega)$, and for every $k>0$. Choosing $\lambda>0$ dividing by $\lambda$, and then letting $\lambda$ tend to zero, we obtain

$$
\int_{\Omega} a(x, u, \nabla u) \nabla T_{k}(u-\Psi) d x+\int_{\Omega} g(x, u) T_{k}[u-\psi] d x \leq \int_{\Omega} f T_{k}(u-\Psi) d x+\int_{\Omega} F(u) \nabla T_{k}(u-\Psi) d x .
$$

for $\lambda<0$, dividing by $\lambda$, and then letting $\lambda$ tend to zero, we obtain

$\int_{\Omega} a(x, u, \nabla u) \nabla T_{k}(u-\Psi) d x+\int_{\Omega} g(x, u) T_{k}[u-\psi] d x \geq \int_{\Omega} f T_{k}(u-\Psi) d x+\int_{\Omega} F(u) \nabla T_{k}(u-\Psi) d x$.

Combining (3.16) and (3.17), we conclude the following equality :

$\int_{\Omega} a(x, u, \nabla u) \nabla T_{k}(u-\Psi) d x+\int_{\Omega} g(x, u) T_{k}[u-\psi] d x=\int_{\Omega} f T_{k}(u-\Psi) d x+\int_{\Omega} F(u) \nabla T_{k}(u-\Psi) d x$.

This completes the proof of Lemma 3.2.

\subsection{Proof of Theorem 3.1.}


3.3.1. Approximate problem and a priori estimate. For $n \in \mathbb{N}$, define $f_{n}:=T_{n}(f), F_{n}=F\left(T_{n}\right)$. Let $u_{n}$ be solution in $W_{0}^{1} L_{\varphi}(\Omega)$ of the problem

$$
\left\{\begin{array}{cl}
-\operatorname{div}\left(a\left(x, u_{n}, \nabla u_{n}\right)\right)+g_{n}\left(x, u_{n}\right)=f_{n}-\operatorname{div} F_{n}\left(u_{n}\right) & \text { in } \Omega \\
u_{n}=0 & \text { on } \partial \Omega,
\end{array}\right.
$$

where,

$$
g_{n}(x, s)=\frac{g(x, s)}{1+\frac{1}{n}|g(x, s)|},
$$

which exists thanks to ([26],Proposition 1, Remark 2). Choosing $T_{k}\left(u_{n}\right)$ as test function in (3.19), we have

$$
\int_{\Omega} a\left(x, u_{n}, \nabla u_{n}\right) \nabla T_{k}\left(u_{n}\right) d x+\int_{\Omega} g_{n}\left(x, u_{n}\right) T_{k}\left(u_{n}\right) d x=\int_{\Omega} f_{n} T_{k}\left(u_{n}\right) d x+\int_{\Omega} F_{n}\left(u_{n}\right) \nabla T_{k}\left(u_{n}\right) d x,
$$

We claim that:

$$
\int_{\Omega} F_{n}\left(u_{n}\right) \nabla T_{k}\left(u_{n}\right) d x=0
$$

using $\nabla T_{k}\left(u_{n}\right)=\nabla u_{n \chi\left\{\left|u_{n}\right| \leq k\right\}}$, define $\Theta(t)=F_{n}(t) \chi\{t \mid \leq k\}$, and $\tilde{\Theta}(t)=\int_{0}^{t} \Theta(\tau) d \tau$,

We have by Lemma $2.13 \tilde{\Theta}\left(u_{n}\right) \in\left(W_{0}^{1} L_{\varphi}(\Omega)\right)^{N}$

$$
\begin{gathered}
\int_{\Omega} F_{n}\left(u_{n}\right) \nabla T_{k}\left(u_{n}\right) d x=\int_{\Omega} F_{n}\left(u_{n}\right) \chi\left\{\left|u_{n}\right| \leq k\right\} \nabla u_{n} d x \\
=\int_{\Omega} \Theta\left(u_{n}\right) \nabla u_{n} d x=\int_{\Omega} \operatorname{div}\left(\tilde{\Theta}\left(u_{n}\right)\right) d x=0
\end{gathered}
$$

(by 2.13) which proves the claim.

Using $\nabla T_{k}\left(u_{n}\right)=\nabla u_{n \chi\left\{\left|u_{n}\right| \leq k\right\}}$ and thanks to assumption (1.4), and since $g_{n}\left(x, u_{n}\right) T_{k}\left(u_{n}\right) \geq$ 0 , we obtain

$$
\int_{\Omega} a\left(x, u_{n}, \nabla u_{n}\right) \nabla T_{k}\left(u_{n}\right) d x \geq \int_{\Omega} \varphi\left(x, \lambda_{1}\left|\nabla T_{k}\left(u_{n}\right)\right|\right) d x
$$

then

$$
\int_{\Omega} \varphi\left(x, \lambda_{1}\left|\nabla T_{k}\left(u_{n}\right)\right|\right) d x \leq k\|f\|_{L^{1}(\Omega)} .
$$

Then

$$
\int_{\Omega} \varphi\left(x, \lambda_{1}\left|\nabla T_{k}\left(u_{n}\right)\right|\right) d x \leq C_{1} k
$$

where $C_{1}$ is a constant independently of $n$. 
3.3.2. Locally convergence of $u_{n}$ in measure. Taking $\lambda\left|T_{k}\left(u_{n}\right)\right|$ in (3.19) and using (3.23), one has

$$
\int_{\Omega} \varphi\left(x, \lambda_{1} \frac{\left|\nabla T_{k}\left(u_{n}\right)\right|}{\lambda}\right) d x \leq \int_{\Omega} \varphi\left(x, \lambda_{1}\left|\nabla T_{k}\left(u_{n}\right)\right|\right) d x \leq C_{1} k .
$$

Then we deduce by using (3.24), that

$$
\begin{aligned}
\operatorname{meas}\left\{\left|u_{n}\right|>k\right\} & \leq \frac{1}{\inf _{k} \varphi\left(x, \frac{k}{\lambda}\right)} \int_{\left\{\left|u_{n}\right|>k\right\}} \varphi\left(x, \frac{\left|u_{n}(x)\right|}{\lambda}\right) d x \\
& \leq \frac{1}{\inf _{k} \varphi\left(x, \frac{k}{\lambda}\right)} \int_{\Omega} \varphi\left(x, \frac{1}{\lambda}\left|T_{k}\left(u_{n}\right)\right|\right) d x \\
& \leq \frac{C_{1} k}{\inf _{k} \varphi\left(x, \frac{k}{\lambda}\right)} \quad \forall n, \quad \forall k \geq 0 .
\end{aligned}
$$

For any $\beta>0$, we have

$$
\operatorname{meas}\left\{\left|u_{n}-u_{m}\right|>\beta\right\} \leq \operatorname{meas}\left\{\left|u_{n}\right|>k\right\}+\operatorname{meas}\left\{\left|u_{m}\right|>k\right\}+\operatorname{meas}\left\{\left|T_{k}\left(u_{n}\right)-T_{k}\left(u_{m}\right)\right|>\beta\right\},
$$

and so that

$$
\operatorname{meas}\left\{\left|u_{n}-u_{m}\right|>\beta\right\} \leq \frac{2 C_{1} k}{\inf _{x \in \Omega} \varphi\left(x, \frac{k}{\lambda}\right)}+\operatorname{meas}\left\{\left|T_{k}\left(u_{n}\right)-T_{k}\left(u_{m}\right)\right|>\beta\right\}
$$

By using (3.23) and Poincaré inequality in musielak-Orlicz-Sobolev spaces (Corollary 2.3), we deduce that $\left(T_{k}\left(u_{n}\right)\right)$ is bounded in $W_{0}^{1} L_{\varphi}(\Omega)$, and then there exists $\omega_{k} \in W_{0}^{1} L_{\varphi}(\Omega)$ such that $T_{k}\left(u_{n}\right) \rightarrow \omega_{k}$ weakly in $W_{0}^{1} L_{\varphi}(\Omega)$ for $\sigma\left(\Pi L_{\varphi}, \Pi E_{\bar{\varphi}}\right)$; strongly in $E_{\bar{\varphi}}(\Omega)$ and a.e. in $\Omega$.

Consequently, we can assume that $\left(T_{k}\left(u_{n}\right)\right)_{n}$ is a Cauchy sequence in measure in $\Omega$.

Let $\varepsilon>0$, then by (3.26) and the fact that $\frac{2 C_{1} k}{\inf _{x \in \Omega} \varphi\left(x, \frac{k}{\lambda}\right)} \rightarrow 0$ as $k \rightarrow+\infty$ there exists some $k=k(\varepsilon)>0$ such that

$$
\operatorname{meas}\left\{\left|u_{n}-u_{m}\right|>\lambda\right\}<\varepsilon, \quad \text { for all } n, m \geq h_{0}(k(\varepsilon), \lambda) \text {. }
$$

This proves that $u_{n}$ is a Cauchy sequence in measure, thus, $u_{n}$ converges almost everywhere to some measurable function $u$. Finally, there exist a subsequence of $\left\{u_{n}\right\}_{n}$, still indexed by $n$, and a function $u \in W_{0}^{1} L_{\varphi}(\Omega)$ such that

$$
\left\{\begin{array}{c}
u_{n} \rightarrow u \quad \text { weakly in } W_{0}^{1} L_{\varphi}(\Omega) \text { for } \sigma\left(\Pi L_{\varphi}, \Pi E_{\bar{\varphi}}\right) \\
u_{n} \longrightarrow u \quad \text { strongly in } E_{\varphi}(\Omega) \text { and a.e. in } \Omega
\end{array}\right.
$$


3.3.3. Equi-integrability of nonlinearities. we need to prove that

$$
g_{n}\left(x, u_{n}\right) \rightarrow g(x, u) \text { strongly in } L^{1}(\Omega)
$$

in particular it is enough to prove the equi-integrable of $g_{n}\left(x, u_{n}\right)$ to this purpose. We take $T_{l+1}\left(u_{n}\right)-T_{l}\left(u_{n}\right)$ as test function in (3.19), we obtain

$$
\begin{gathered}
\int_{\Omega} a\left(x, u_{n}, \nabla u_{n}\right) \nabla\left(T_{l+1}\left(u_{n}\right)-T_{l}\left(u_{n}\right)\right) d x+\int_{\Omega} g_{n}\left(x, u_{n}\right)\left(T_{l+1}\left(u_{n}\right)-T_{l}\left(u_{n}\right)\right) d x \\
=\int_{\Omega} f\left(T_{l+1}\left(u_{n}\right)-T_{l}\left(u_{n}\right)\right) d x
\end{gathered}
$$

which implies that

$$
\begin{aligned}
& \int_{\left\{l \leq\left|u_{n}\right| \leq l+1\right\}} a\left(x, u_{n} . \nabla u_{n}\right), \nabla u_{n} d x+\int_{\left\{\left|u_{n}\right| \geq l+1\right\}}\left|g_{n}\left(x, u_{n}\right)\right| d x \\
& \quad \leq c \int_{\left\{\left|u_{n}\right| \geq l\right\}}|f| d x .
\end{aligned}
$$

Thus by (1.4), we have

$$
\int_{\left\{\left|u_{n}\right| \geq l+1\right\}}\left|g_{n}\left(x, u_{n}\right)\right| d x \leq c \int_{\left\{\left|u_{n}\right| \geq l\right\}}\left|f_{n}\right| d x .
$$

Let $\varepsilon>0$, then there exist $l(\varepsilon) \geq 1$ such that

$$
\int_{\left\{\left|u_{n}\right|>l(\varepsilon)\right\}}\left|g_{n}\left(x, u_{n}\right)\right| d x \leq \frac{\varepsilon}{2},
$$

For any measurable subset $E \subset \Omega$, we have

$$
\begin{aligned}
\int_{E}\left|g_{n}\left(x, u_{n}\right)\right| d x & \leq \int_{E \cap\left\{\left|u_{n}\right| \leq l(\varepsilon)\right\}}\left|g_{n}\left(x, u_{n}\right)\right| d x+\int_{E \cap\left\{\left|u_{n}\right|>l(\varepsilon)\right\}}\left|g_{n}\left(x, u_{n}\right)\right| d x \\
& \leq \int_{E}\left|h_{l(\varepsilon)}(x)\right| d x+\int_{E \cap\left\{\left|u_{n}\right|>l(\varepsilon)\right\}}\left|g_{n}\left(x, u_{n}\right)\right| d x
\end{aligned}
$$

In view to $(1.6)$ there exist $\eta(\varepsilon)>0$ such that

$$
\int_{E}\left|h_{l(\varepsilon)}(x)\right| d x \leq \frac{\varepsilon}{2},
$$

for all $E$ such that meas $(E)<\eta(\varepsilon)$

Finally, by combining (3.29) and (3.30) one easily has $\int_{E}\left|g_{n}\left(x, u_{n}\right)\right| d x \leq \varepsilon$, for all $E$ such that meas $(E)<\eta(\varepsilon)$ 
3.3.4. An intermediate Inequality. In this step, we shall prove that for $\phi \in W_{0}^{1} L_{\varphi}(\Omega)$, we have

$$
\begin{array}{r}
\int_{\Omega} a\left(x, u_{n}, \nabla \phi\right) \nabla T_{k}\left(u_{n}-\phi\right) d x+\int_{\Omega} g_{n}\left(x, u_{n}\right) T_{k}\left(u_{n}-\phi\right) d x \\
\leq \int_{\Omega} f_{n} T_{k}\left(u_{n}-\phi\right) d x+\int_{\Omega} F_{n} \nabla T_{k}\left(u_{n}-\phi\right) d x
\end{array}
$$

We choose now $T_{k}\left(u_{n}-\phi\right)$ as test function in (3.19), with $\phi$ in $W_{0}^{1} L_{\varphi}(\Omega)$, we obtain

$$
\begin{aligned}
\int_{\Omega} a\left(x, u_{n}, \nabla u_{n}\right) & \nabla T_{k}\left(u_{n}-\phi\right) d x+\int_{\Omega} g_{n}\left(x, u_{n}\right) T_{k}\left(u_{n}-\phi\right) d x \\
= & \int_{\Omega} f_{n} T_{k}\left(u_{n}-\phi\right) d x+\int_{\Omega} F_{n} \nabla T_{k}\left(u_{n}-\phi\right) d x
\end{aligned}
$$

Adding and substracting the term $\int_{\Omega} a\left(x, u_{n}, \nabla \phi\right), \nabla T_{k}\left(u_{n}-\phi\right) d x$ i.e.,

$$
\begin{gathered}
\int_{\Omega} a\left(x, u_{n}, \nabla u_{n}\right) \nabla T_{k}\left(u_{n}-\phi\right) d x+\int_{\Omega} a\left(x, u_{n}, \nabla \phi\right) \nabla T_{k}\left(u_{n}-\phi\right) d x \\
-\int_{\Omega} a\left(x, u_{n}, \nabla \phi\right) \nabla T_{k}\left(u_{n}-\phi\right) d x+\int_{\Omega} g_{n}\left(x, u_{n}\right) T_{k}\left(u_{n}-\phi\right) d x= \\
\int_{\Omega} f_{n} T_{k}\left(u_{n}-\phi\right) d x+\int_{\Omega} F_{n} \nabla T_{k}\left(u_{n}-\phi\right) d x
\end{gathered}
$$

Thanks to assumption (1.3) and the definition of truncation function, we have

$$
\int_{\Omega}\left(a\left(x, u_{n}, \nabla u_{n}\right)-a\left(x, u_{n}, \nabla \phi\right)\right) \nabla T_{k}\left(u_{n}-\phi\right) d x \geq 0 .
$$

Combining (3.35) and (3.36), we obtain (3.31).

3.3.5. Passing to the limit. We shall prove that for $\phi \in W_{0}^{1} L_{\varphi}(\Omega)$, we have

$$
\int_{\Omega} a(x, u, \nabla \phi) \nabla T_{k}(u-\phi) d x+\int_{\Omega} g_{n}\left(x, u_{n}\right) T_{k}\left(u_{n}-\phi\right) d x \leq \int_{\Omega} f T_{k}(u-\phi) d x+\int_{\Omega} F \nabla T_{k}(u-\phi) d x .
$$

Firstly, we claim that

$$
\int_{\Omega} a\left(x, u_{n}, \nabla \phi\right) \nabla T_{k}\left(u_{n}-\phi\right) d x \rightarrow \int_{\Omega} a(x, u, \nabla \phi) \nabla T_{k}(u-\phi) d x \text { as } n \rightarrow+\infty .
$$

Since $T_{M}\left(u_{n}\right) \rightarrow T_{M}(u)$ weakly in $W_{0}^{1} L_{\varphi}(\Omega)$, with $M=k+\|\phi\|_{\infty}$, then

$$
T_{k}\left(u_{n}-\phi\right) \rightarrow T_{k}(u-\phi) \text { in } W_{0}^{1} L_{\varphi}(\Omega)
$$

which gives

$$
\frac{\partial T_{k}}{\partial x_{i}}\left(u_{n}-\phi\right) \rightarrow \frac{\partial T_{k}}{\partial x_{i}}(u-\phi) \text { weakly in } L_{\varphi}(\Omega) \quad \forall i=1, . ., N
$$


Show that

$$
a\left(x, T_{M}\left(u_{n}\right), \nabla \phi\right) \rightarrow a\left(x, T_{M}(u), \nabla \phi\right) \text { strongly in }\left(L_{\bar{M}}(\Omega)\right)^{N}
$$

Thanks to assumption (1.2), we obtain

$$
\left|a_{i}\left(x, T_{M}\left(u_{n}\right), \nabla \phi\right)\right| \leq\left|\phi_{i}(x)\right|+K_{i} \bar{P}^{-1}\left(\varphi\left(x, c_{2}\left|T_{M}\left(u_{n}\right)\right|\right)\right)+K_{i} \bar{\varphi}^{-1} \varphi\left(x, c_{1}|\nabla \phi|\right),
$$

with $\beta$ and $\mu$ are positive constants. Since $T_{M}\left(u_{n}\right) \rightarrow T_{M}(u)$ weakly in $W_{0}^{1} L_{\varphi}(\Omega)$ and $W_{0}^{1} L_{\varphi}(\Omega) \hookrightarrow \hookrightarrow$ $L_{\bar{\varphi}}(\Omega)$, then $T_{M}\left(u_{n}\right) \rightarrow T_{M}(u)$ strongly in $L_{\varphi}(\Omega)$ and a.e. in $\Omega$, hence

$$
\left|a\left(x, T_{M}\left(u_{n}\right), \nabla \phi\right)\right| \rightarrow\left|a\left(x, T_{M}(u), \nabla \phi\right)\right| \text { a.e. in } \Omega .
$$

and

$$
\begin{gathered}
\left|\phi_{i}(x)\right|+K_{i} \bar{P}^{-1}\left(\varphi\left(x, c_{2}\left|T_{M}\left(u_{n}\right)\right|\right)\right)+K_{i} \bar{\varphi}^{-1} \varphi\left(x, c_{1}|\nabla \phi|\right) \rightarrow \\
\left|\phi_{i}(x)\right|+K_{i} \bar{P}^{-1}\left(\varphi\left(x, c_{2}\left|T_{M}(u)\right|\right)\right)+K_{i} \bar{\varphi}^{-1} \varphi\left(x, c_{1}|\nabla \phi|\right),
\end{gathered}
$$

a.e. in $\Omega$. Then, By Vitali's theorem, we deduce that

$$
a\left(x, T_{M}\left(u_{n}\right), \nabla \phi\right) \rightarrow a\left(x, T_{M}(u), \nabla \phi\right) \text { strongly in }\left(L_{\bar{\varphi}}(\Omega)\right)^{N}, \text { as } n \rightarrow \infty \text {. }
$$

Combining (3.38) and (3.39), we obtain

$$
\int_{\Omega} a\left(x, u_{n}, \nabla \phi\right) \nabla T_{k}\left(u_{n}-\phi\right) d x \rightarrow \int_{\Omega} a(x, u, \nabla \phi) \nabla T_{k}(u-\phi) d x \text { as } n \rightarrow+\infty .
$$

Secondly, we show that

$$
\int_{\Omega} f_{n} T_{k}\left(u_{n}-\phi\right) d x \rightarrow \int_{\Omega} f T_{k}(u-\phi) d x
$$

and

$$
\int_{\Omega} F_{n} \nabla T_{k}\left(u_{n}-\phi\right) d x \rightarrow \int_{\Omega} F \nabla T_{k}(u-\phi) d x
$$

We have $f_{n} T_{k}\left(u_{n}-\phi\right) \rightarrow f T_{k}(u-\phi)$ a.e. in $\Omega$ and $\left|f T_{k}\left(u_{n}-\phi\right)\right| \leq k|f|$, and $F_{n} \nabla T_{k}\left(u_{n}-\phi\right) \rightarrow F \nabla T_{k}(u-\phi)$ a.e. in $\Omega$, and $\left|F \nabla T_{k}\left(u_{n}-\phi\right)\right| \leq k|F|$

then by using Vitali's theorem, we obtain (3.41)and (3.42) .

Similarly thanks to (3.28) we can show that

$$
\int_{\Omega} g_{n}\left(x, u_{n}\right) T_{k}\left(u_{n}-\phi\right) d x \rightarrow \int_{\Omega} g(x, u) T_{k}(u-\phi) d x \text { as } n \rightarrow \infty .
$$


Thanks to (3.40), (3.41)and (3.42) allow to pass to the limit in the inequality (3.31), so that $\forall \phi \in W_{0}^{1} L_{\varphi}(\Omega)$, we deduce

$$
\int_{\Omega} a(x, u, \nabla \phi) \nabla T_{k}(u-\phi) d x+\int_{\Omega} g(x, u) T_{k}(u-\phi) d x \leq \int_{\Omega} f T_{k}(u-\phi) d x+\int_{\Omega} F \nabla T_{k}(u-\phi) d x .
$$

In view of main Lemma, we can deduce that $u$ is an entropy solution of the problem (1.1). This completes the proof of Theorem 3.1 .

\section{REFERENCES}

[1] R. Adams, Sobolev spaces, Academic Press, New York, 1975.

[2] Y. Akdime, E. Azroul, M. Rhoudaf, Existence of T-solution for degenerated problem via Minty's lemma, Acta. Math. Sin.-English Ser. 24 (2008), 431-438.

[3] E.H. Azroul, A. Barbara, M. El Lekhlifi, M. Rhoudaf, T-p(x)-solutions for nonlinear elliptic equations with an $L^{1}$-dual datum, Appl. Math. (Warsaw). 39 (2012), 339-364.

[4] M. Ben Cheikh Ali, O. Guibe, Nonlinear and non-coercive elliptic problems with integrable data, Adv. Math. Sci. Appl. 16 (2006), 275-297.

[5] M. Ait Khellou, A. Benkirane, S.M. Douiri, Some properties of Musielak spaces with only the log-Hölder continuity condition and application, Ann. Funct. Anal. 11 (2020), 1062-1080.

[6] P. Bénilan, L. Boccardo, T. Gallouët, R. Gariepy, M. Pierre, J.L. Vázquez; An $L^{1}$-theory of existence and uniqueness of solutions of nonlinear elliptic equations, Ann. Scuola Norm. Sup. Pisa Cl. Sci. 4 (1995), 241-273.

[7] A. Benkirane, A. Elmahi, Almost everywhere convergence of the gradient of solutions to elliptic equations in Orlicz spaces, Nonlinear Anal. Theory Meth. Appl. 28(11)(1997), 1769-1784.

[8] A. Benkirane, A. Elmahi, An existence theorem for a strongly nonlinear elliptic problem in Orlicz spaces, Nonlinear Anal. Theory Meth. Appl. 36(1) (1999), 11-24.

[9] A. Benkirane and M. Sidi El Vally, Some approximation properties in Musielak-Orlicz- Sobolev spaces, Thai. J. Math. 10 (2012), 371-381.

[10] A. Benkirane, M. Sidi El Vally, Variational inequalities in Musielak-Orlicz-Sobolev spaces, Bull. Belg. Math. Soc. Simon Stevin 21 (2014), 787-811.

[11] A. Benkirane, B. El Haji and M. El Moumni, On the existence of solution for degenerate parabolic equations with singular terms, Pure Appl. Math. Quart. 14 (3-4) (2018), 591-606.

[12] A. Benkirane, B. EL Haji, M. EL Moumni, Strongly nonlinear elliptic problem with measure data in Musielak-Orlicz spaces, Complex Var. Elliptic Equ. (2021), 1-23. https://doi .org/10.1080/17476933. 2021.1882434.

[13] L. Boccardo, L. Orsina, Existence results for dirichlet problems in $L^{1}$ via minty's lemma, Appl. Anal. 76 (2000), 309-317. 
[14] L. Boccardo, Some nonlinear Dirichlet problems in $L^{1}$ involving lower order terms in divergence form, Progress in Elliptic and Parabolic Partial Differential Equations (Capri, 1994 ), Pitman Res. Notes Math. Ser., vol. 350, Longman, Harlow, (1996), 43-57.

[15] H. Brézis, Opérateursmaximaux monotones et semi-groupes de contractions dans les espaces de Hilbert, North Holland, Amsterdam, 1973.

[16] F.E. Browder, Existence theorems for nonlinear partial differential equations, Global Analysis (Proc. Sympos. Pure Math), Vol. XVI, Berkeley, Calif, (1968), Amer. Math. Soc, Providence, R.I., (1970), pp. 1-60.

[17] [1]A. Dall'Aglio, Approximated solutions of equations with $L^{1}$ data. Application to the H-convergence of quasi-linear parabolic equations, Ann. Mat. Pura Appl. 170 (1996), 207-240.

[18] J. Droniou, A. Prignet, Equivalence between entropy and renormalized solutions for parabolic equations with smooth measure data, Nonlinear Differ. Equ. Appl. 14 (2007), 181-205.

[19] R.J. DiPerna, P.L. Lions, On the Cauchy problem for boltzmann equations: global existence and weak stability, Ann. Math. 130 (1989), 321-366.

[20] B. El Haji, M. El Moumni, A. Talha, Entropy solutions for nonlinear parabolic equations in Musielak Orlicz spaces without Delta ${ }_{2}$-conditions, Gulf J. Math. 9 (2020), 1-26.

[21] B. El Haji, M. El Moumni, Entropy solutions of nonlinear elliptic equations with $L^{1}$-data and without strict monotonocity conditions in weighted Orlicz-Sobolev spaces, J. Nonlinear Funct. Anal. 2021 (2021), 8, pp $1-17$.

[22] B. El haji, M. El Moumni, K. Kouhaila, On a nonlinear elliptic problems having large monotonocitywith $L^{1}$-data in weighted Orlicz-Sobolev spaces, Moroccan J. Pure Appl. Anal. 5 (2019), 104-116.

[23] N.E. Amarty, B.E. Haji, M.E. Moumni, Existence of renomalized solution for nonlinear elliptic boundary value problem without $\Delta_{2}$-condition, SeMA. 77 (2020), 389-414.

[24] R. Elarabi, M. Rhoudaf, H. Sabiki, Entropy solution for a nonlinear elliptic problem with lower order term in Musielak-Orlicz spaces, Ricerche Mat. 67 (2018), 549-579.

[25] J.-P. Gossez, Some approximation properties in Orlicz-Sobolev spaces, Stud. Math. 74 (1982), 17-24.

[26] J.-P. Gossez, V. Mustonen, Variational inequalities in Orlicz-Sobolev spaces, Nonlinear Anal.: Theory Meth. Appl. 11 (1987), 379-392.

[27] E. Hewitt, K. Stromberg, Real and Abstract Analysis, Springer, Berlin, (1965).

[28] C. Leone, A. Porretta, Entropy solutions for nonlinear elliptic equations in $L^{1}$, Nonlinear Anal.: Theory Meth. Appl. 32 (1998), 325-334.

[29] P.L. Lions, F. Murat, Sur les solutions renormalisées d'équations elliptiques non linéaires. C.R. Acad. Sci. Paris, (1965).

[30] P.L. Lions, Quelques mèthodes de rèsolution des problèmes aux limites non linèaire, Dunod et Gauthier Villars, Paris, 1969.

[31] G.J. Minty, Monotone (nonlinear) operators in Hilbert space, Duke Math. J. 29 (1962), 341-346.

[32] J. Musielak, Modular spaces and Orlicz spaces, Lecture Notes in Math. Vol. 1034, Springer-Verlag, Berlin, 1983. 
[33] F. Petitta, A.C. Ponce, A. Porretta, Diffuse measures and nonlinear parabolic equations, J. Evol. Equ. 11 (2011), 861-905

[34] F. Petitta, Asymptotic behavior of solutions for parabolic operators of Leray-Lions type and measure data. Adv. Differ. Equ. 12 (2007), 867-891.

[35] A. Prignet, Existence and uniqueness of "entropy" solutions of parabolic problems with $L^{1}$ data, Nonlinear Anal. Theory Meth. Appl. 28 (1997), 1943-1954.

[36] A. Porretta, S. Segura de León, Nonlinear elliptic equations having a gradient term with natural growth, J. Math. Pures Appl. 85 (2006), 465-492.

[37] A. Porretta, Existence results for nonlinear parabolic equations via strong convergence of truncations, Ann. Mat. Pura Appl. 177 (1999), 143-172.

[38] S. Polidoro, M.A. Ragusa, Harnack inequality for hypoelliptic ultraparabolic equations with a singular lower order term, Rev. Mat. Iberoamericana, 24 (2008), 1011-1046.

[39] M.A. Ragusa, Elliptic boundary value problem in vanishing mean oscillation hypothesis, Comment. Math. Univ. Carolin, 40 (1999), 651-663.

[40] W. Rudin, Real and Complex Analysis, 3rd ed., McGraw-Hill, New York, (1974). 